\title{
Intractable stokes adams attack with myriad of ventricular arrythmias masquerading acute myocarditis
}

\author{
Mandeep Kaur*1, Prema Bezwada ${ }^{2}$, Cesar E. Ayala-Rodriguez ${ }^{3}$ \\ ${ }^{1}$ Department of Internal Medicine, The Brooklyn Hospital Center, New York, United States \\ ${ }^{2}$ Department of Cardiology, Mount Sinai, New York, United States \\ ${ }^{3}$ Department of Cardiology, The Brooklyn Hospital Center, Brooklyn, New York, United States
}

Received: May 22, 2019

DOI: $10.5430 /$ crim.v6n4p9
Accepted: August 12, 2019

Online Published: September 24, 2019

\begin{abstract}
Convulsive syncope can be difficult to distinguish from seizures at times. Misdiagnosis can result in unnecessary, and usually unsuccessful, treatment with antiepileptic drugs. A detailed history taking and physical examination along with continuous cardiac monitoring can help in making the correct diagnosis. Our patient was eventually found to have viral myocarditis. More detailed studies for the role of noninvasive cardiac testing such as Cardiac MRI (CMR) and newer treatment modalities such as anti-thymocyte immunoglobulin are required for the management of Viral Myocarditis.
\end{abstract}

Key Words: Stokes Adams, Ventricular tachycardia, Myocarditis

\section{INTRODUCTION}

Differentiating Cardiovascular Convulsive syncope from Seizure has always been a dilemma. ${ }^{[1,2]}$ We describe a case of a previously healthy young woman presenting with a sudden onset of intractable Stokes Adam Attack. Vasovagal syncope is the most likely etiology of syncope in young women. ${ }^{[1]}$ However detailed history taking in our patient revealed a prodrome of viral illness. Later found to have myocarditis on cardiac MRI. Myocarditis can have variable presentation. ${ }^{[1,3]}$ This case depicts life threatening arrhythmias that present as seizure like episodes. The arrythmias caused as a sequela of myocarditis.

\section{Case presentation}

A 32-year-old female with no pertinent past medical history was brought to the Emergency Department after a cardiac arrest at work. As per coworkers she suddenly collapsed and had seizure like activity. She was found to be pulseless, so three rounds of CPR were performed and four shocks were delivered. Return of spontaneous circulation was achieved. She reverted to full consciousness spontaneously within minutes without confusion or cognitive deficits. On further enquiry she admitted a one-week history of fever and cough. She was evaluated in an urgent care Center and was sent home with Pseudoephedrine. En route to the hospital she was noted to have a BP of $90 / 60 \mathrm{mmHg}$ and the cardiac monitor revealed Ventricular tachycardia (VT) (see Figure 1). She then had an episode of myoclonus and became unresponsive, went into asystole briefly then polymorphic VT then monomorphic VT and finally into sinus tachycardia. Per EMS this lasted for about $15 \mathrm{sec}$. In the Emergency department, cardiac monitor showed non-sustained polymorphic ventricular tachycardia and then a sustained period of

\footnotetext{
*Correspondence: Mandeep Kaur; Email: kaurmandeep1880@ gmail.com; Address: The Brooklyn Hospital Center 121 De Kalb Avenue, Brooklyn, New York, NY 11201, United States.
} 
bigeminy quickly developing into polymorphic ventricular tachycardia with tonic movements of her arms and face and was pulseless (see Figure 2). CPR and defibrillation were performed with resolution to sinus rhythm. She quickly regained consciousness. Total time of pulselessness was less than 30 seconds. Cardiac monitoring revealed frequent Premature Ventricular Contractions (PVC). Point of care ultrasound was performed bedside, which showed mildly dilated left atrium and left ventricle with reduced left ventricular ejection fraction (see Figure 3). She was overdrive paced for a day. On telemonitoring, she was found to be in sinus rhythm with PVC . IV Amiodarone was also given to control ventricular arrhythmias.

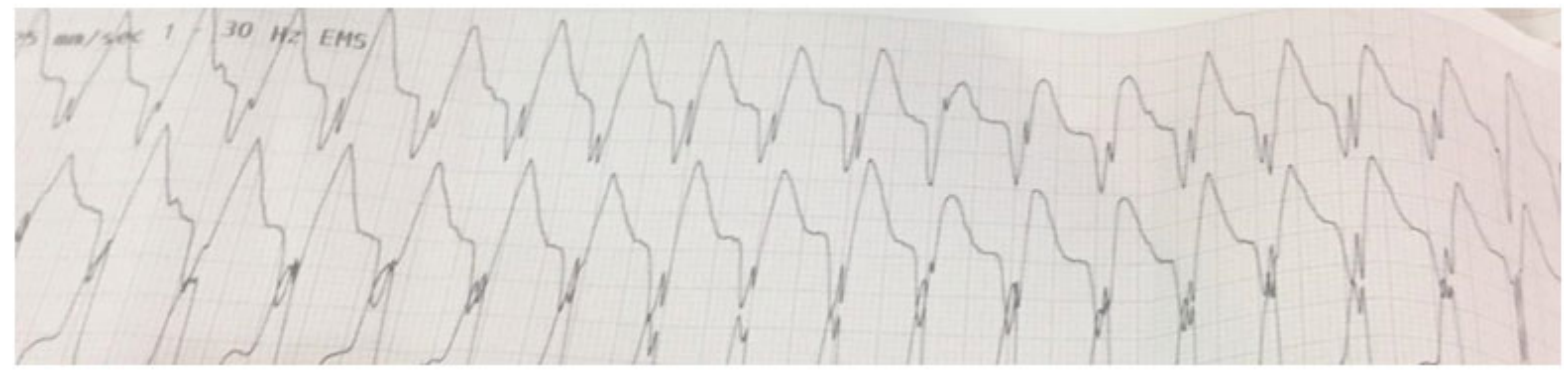

Figure 1. Electrocardiogram recorded by Emergency Medical Services showing Monomorphic Ventricular Tachycardia

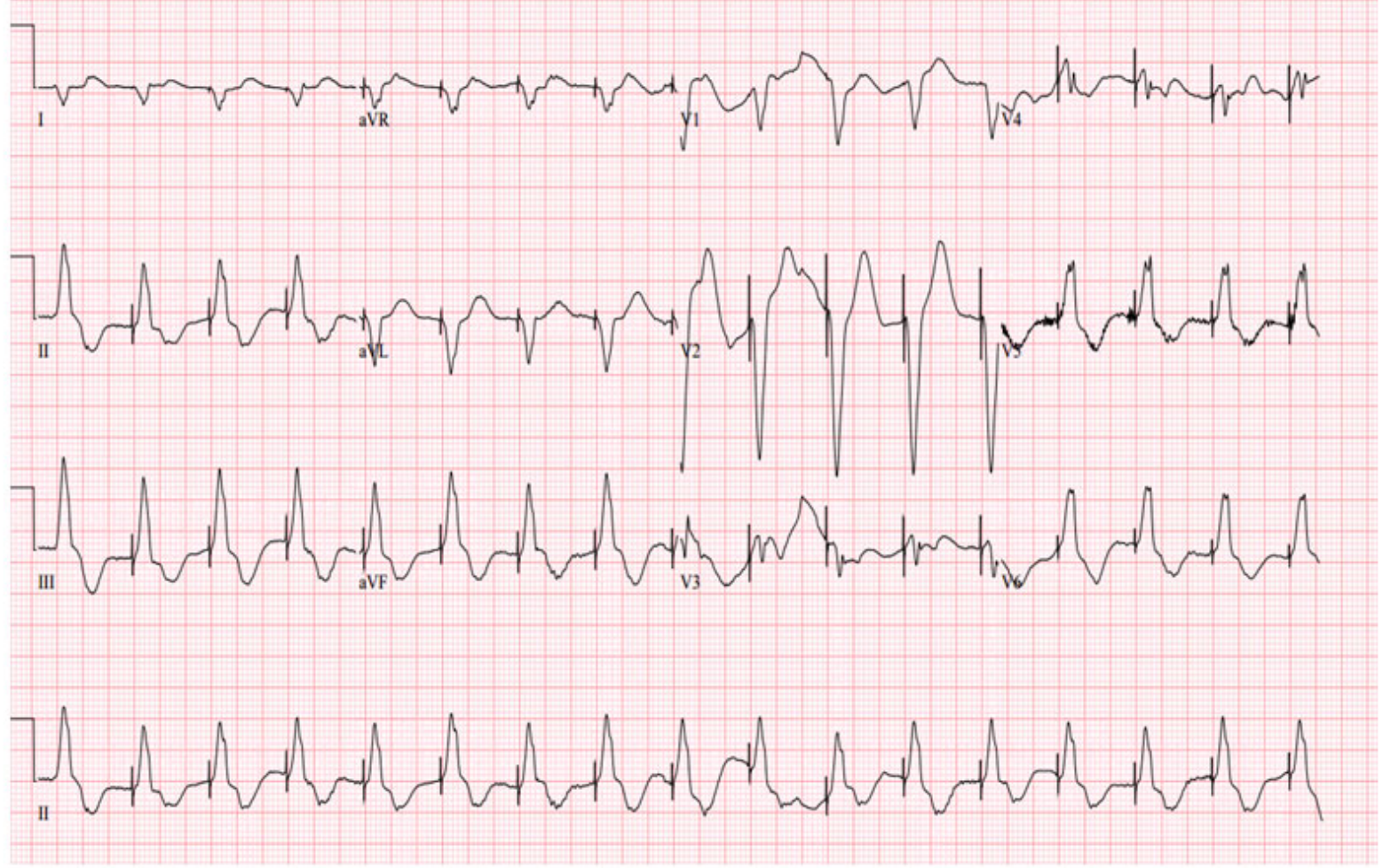

Figure 2. EKG showing ventricular paced rhythm

On day 2, TVP was removed and patient was transferred to a tertiary care center for a cardiac MRI. Cardiac MRI revealed Global myocardial SI increase in T2-weighted images and Increased global myocardial early gadolinium enhancement ratio between myocardium and skeletal muscle in gadolinium-enhanced T1-weighted image. A Subcutaneous Implantable Cardioverter Defibrillator (ICD) was placed for secondary prevention of arrhythmias. Patient was discharged with close follow up. 


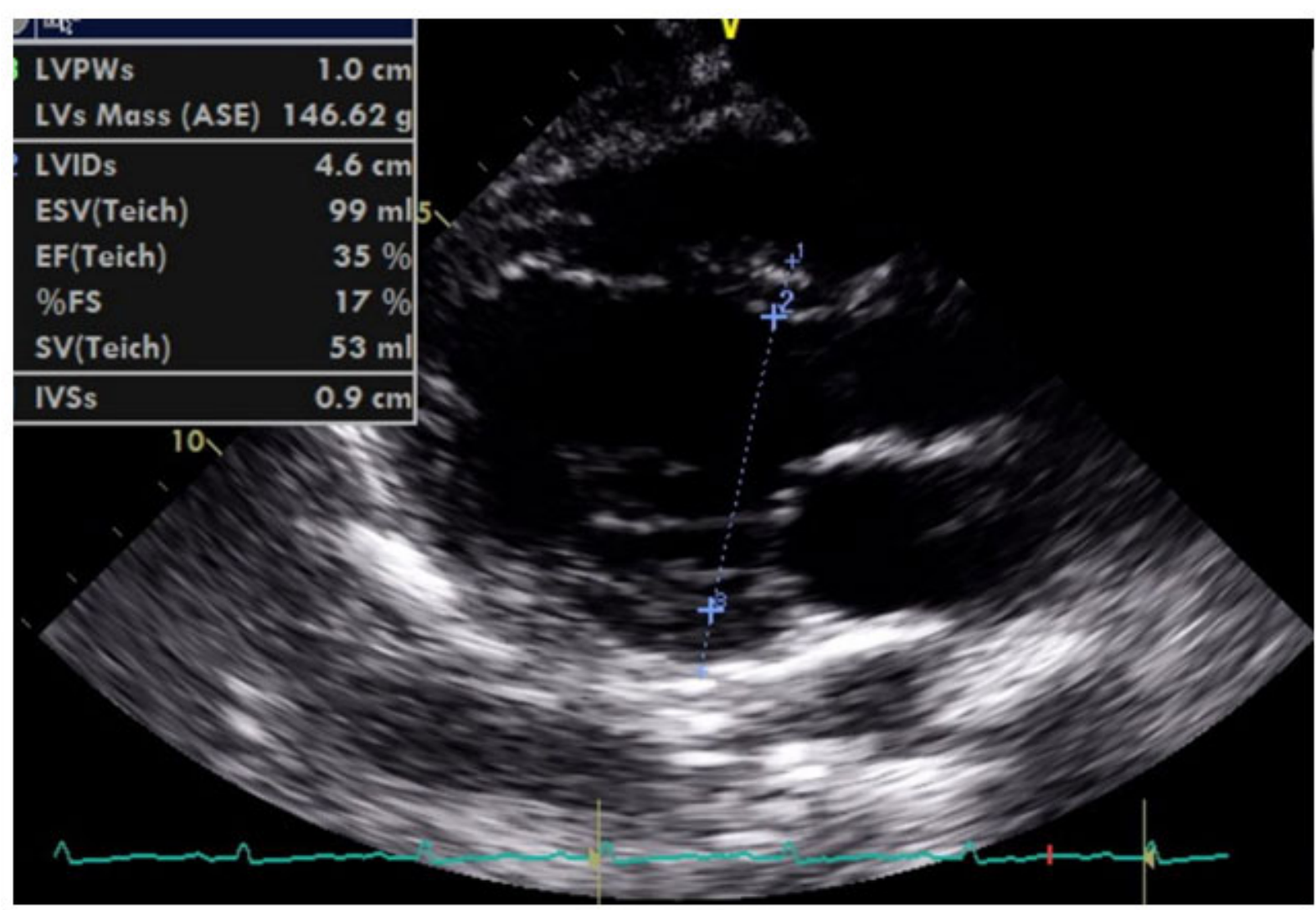

Figure 3. Transthoracic Echocardiogram showing Dilated Left Ventricle with reduced Ejection Fraction. Left Ventricular Ejection Fraction: 35 percent. Dilated Left Atrium with diameter of $2.6 \mathrm{~cm}$.

\section{Discussion}

This case describes a patient with classic Stokes Adam attack associated with a myriad of ventricular arrhythmias in the setting of Acute viral myocarditis. The Stokes-Adams attack, a form of cardiovascular syncope, classically described as an abrupt, transient loss of consciousness due to a sudden but pronounced decrease in the cardiac output, which is caused by a paroxysmal shift in hemodynamics. ${ }^{[1,2]}$ Our patient presented with generalized jerking and myoclonic movements with loss of consciousness and loss of motor tone. The differential diagnosis of seizures versus syncope was considered. The loss of muscle tone (atonia) resulting in sudden collapse, the absence of post ictal phase, prompt return of consciousness favors syncope over seizure in our patient. ${ }^{[1,4]}$ The onset of syncope correlated with the detection of arrhythmias causing hypotension on the cardiac monitor. Stokes-Adams attacks are related to paroxysmal or chronic atrioventricular (AV) block (50\%-60\%), sinoatrial (SA) block (30\%-40\%) or paroxysmal tachycardia or fibrillation (0-5\%). Diagnosis is based on EKG recording during fainting. Pacemaker implantation should be performed in Stokes-Adams syndrome, as oral drug treatment is ineffective. ${ }^{[5]}$ Myocarditis presents in many different ways, Published by Sciedu Press ranging from mild symptoms of chest pain and palpitations associated with transient ECG changes to life-threatening cardiogenic shock and ventricular arrhythmia. ${ }^{[3,6]}$ Treatment of myocarditis consists of both specific therapy aimed at the cause of the myocarditis and supportive treatment to control Heart failure and arrhythmias. ${ }^{[7-9]}$ The diagnostic evaluation of patients with suspected myocarditis includes history and physical examination to evaluate for symptoms and signs of myocarditis and heart failure (HF). ${ }^{[3,8]}$ Initial laboratory testing including a serum troponin levels, and chest X Ray was non diagnostic. Cardiac monitoring revealed myriad of ventricular arrhythmias. An echocardiogram revealed decreased ejection fraction and dilated cardiomyopathy. Coronary angiography was deferred as our patient did not have high-risk features for ischemic heart disease. Cardiovascular magnetic resonance (CMR) imaging was performed as patient had ventricular dysfunction, without a clear cause such as ischemic heart disease. ${ }^{[8,10]}$ CMR provided a supportive evidence of myocarditis. The decision on whether to proceed with Endomyocardial Biopsy (EMB) is based upon the likelihood that EMB will significantly impact patient management. ${ }^{[8]}$ Emphasis is placed on defining viral and autoimmune heart disease with the use of methodologies

Published by Sciedu Press 
available in the 21 st century. Previously used Dallas criteria has questionable utility. A new criterion, which should include clinical presentation, histopathology, immunohistochemistry, viral polymerase chain reaction, cardiac antibody assessment, and imaging results is required for diagnosis of viral myocarditis. ${ }^{[11]}$

It is a common case scenario where patients are dismissed from urgent care centers with common cold remedies to have to return with life threatening Myocarditis/Bacterial superinfections. ${ }^{[7]}$ Unfortunately, we do not have any predictive methods to triage these patients on initial presentation at urgent care centers. Role of CMR and use of IVIG therapy in myocarditis need further consideration. ${ }^{[8,12]}$

\section{Conclusion}

Differentiating syncope from seizures can be difficult at times especially when syncope is accompanied by anoxia-induced involuntary movement. Clinical features may not always be reliable. In cases where diagnosis is uncertain, circulatory and cardiac causes should always be kept in mind and video-
EEG with simultaneous cardiac recordings are mandatory for accuracy of diagnosis. ${ }^{[1,2,4]}$ Literature reveals various presentations of Stokes Adam Attack. A myriad of arrhythmias are associated with it. AV blocks are more commonly associated than Ventricular arrhythmias. ${ }^{[13]}$ Temporary venous pacing is the mainstay of treatment. Cardiovascular magnetic resonance $(\mathrm{CMR})$ has become the primary tool for noninvasive assessment of myocardial inflammation in patients with suspected myocarditis. ${ }^{[3]}$ Current standard treatment of viral myocarditis is supportive care, although immunomodulatory therapies, such as steroids and intravenous immunoglobulin, are often used. A systematic review concluded that there are insufficient data from methodologically strong studies to recommend routine IVIG therapy in patients with acute myocarditis. However, there are no controlled randomized data on IVIG in pediatric or adult patients with acute or chronic myocarditis or Dilated cardiomyopathy with biopsy-proven viral or autoreactive myocardial inflammation. ${ }^{[9]}$

\section{CONFLicts OF InTEREST Disclosure}

The author declares no conflict of interest

\section{REFERENCES}

[1] James Webb MD, Brit Long MD, Alex Koyfman MD. An Emergency Medicine-Focused Review of Seizure Mimics. J Emerg Med. 2017; 52(5): 645-53. PMid:28007363. https://doi.org/10.1016/j. jemermed.2016.11.002

[2] Ozkara C, Metin B, Kucukoglu S. Convulsive syncope: a condition to be differentiated from epilepsy. Epileptic Disord. 2009 Dec; 11(4): 315-9. PMid:20045781. https ://doi .org/10.1684/epd. 2009 .0281

[3] Alida L. P. Caforio, Sabine Pankuweit, Eloisa Arbustini, Cristina Basso, Juan Gimeno-Blanes et al. Current state of knowledge on aetiology, diagnosis, management, and therapy of myocarditis: a position statement of the European Society of Cardiology Working Group on Myocardial and Pericardial Diseases. Eur Heart J. 2013 September; 34(33): 2636-48. PMid:23824828. https://doi.org/ 10.1093/eurheartj/eht210

[4] Robert Sheldon. How to Differentiate Syncope from Seizure, Cardiology Clinics. 2015; 33(3): 377-385. PMid:26115824. https: //doi.org/10.1016/j.ccl.2015.04.006

[5] Bergfeldt L. Differential diagnosis of cardiogenic syncope and seizure disorders. Heart. 2003 Mar; 89(3): 353-8. PMid:12591858 https://doi.org/10.1136/heart.89.3.353

[6] Donald. A. Dupler. Ventricular Arrhythmia and Stokes-Adams Syndrome: Report of a Case. Circulation. 1953; 7: 585-90. https: //doi.org/10.1161/01.CIR.7.4.585
[7] Maisch, B. Inflammatory cardiomyopathy and myocarditis. Herz. 2017; 42(4): 425-38. PMid:28451703. https://doi .org/10.100 7/s00059-017-4569-y

[8] Cooper LT, McKenna WJ, Yeon SB. Clinical manifestations and diagnosis of myocarditis in adults. 2011; 19: 3 .

[9] Jenna AP, Marc ER, Eva WC, et al. Influenza Myocarditis Treated With Antithymocyte Globulin. Pediatrics. 2018 November; 142(5): e20180884. PMid:30352793. https://doi.org/10.1542/peds .2018-0884

[10] Friedrich MG, Sechtem U, Schulz-Menger J, et al. Cardiovascular magnetic resonance in myocarditis: A JACC White Paper. J Am Coll Cardiol. 2009; 53(17): 1475-1487. PMid:19389557. https : //doi.org/10.1016/j.jacc. 2009.02.007

[11] Baughman KL. Diagnosis of myocarditis: death of Dallas criteria. Circulation. 2006 Jan 31; 113(4): 593-5. PMid:16449736. https://doi.org/10.1161/CIRCULATIONAHA . 105.589663

[12] Kasper DL, Fauci AS, Hauser SL, Longo DL, Jameson J, Loscalzo J. eds. Cardiomyopathies and Myocarditis. In: Harrison's Manual of Medicine, 19e New York, NY: McGraw-Hill. http: //accessmedicine.mhmedical. com. eresources.mssm.e $\mathrm{du} /$ content . aspx?bookid=1820\&sectionid $=127557817$. Accessed April 15, 2019.

[13] Sigurd B, Sandøe E: Management of Stokes-Adams Syndrome. Cardiology. 1990; 77: 195-208. PMid:2272057. https://doi.org/10 $.1159 / 000174601$ 